\title{
The clinical effectiveness of reflectance optical spectroscopy for the in vivo diagnosis of oral lesions
}

\begin{abstract}
Diana V Messadi ${ }^{1}$, Fariba S Younai ${ }^{2}$, Hong-Hu Liu ${ }^{3}$, Gao Guo ${ }^{2}$ and Cun-Yu Wang ${ }^{2}$
Optical spectroscopy devices are being developed and tested for the screening and diagnosis of oral precancer and cancer lesions. This study reports a device that uses white light for detection of suspicious lesions and green-amber light at $545 \mathrm{~nm}$ that detect tissue vascularity on patients with several suspicious oral lesions. The clinical grading of vascularity was compared to the histological grading of the biopsied lesions using specific biomarkers. Such a device, in the hands of dentists and other health professionals, could greatly increase the number of oral cancerous lesions detected in early phase. The purpose of this study is to correlate the clinical grading of tissue vascularity in several oral suspicious lesions using the Identafi ${ }^{\circledR}$ system with the histological grading of the biopsied lesions using specific vascular markers. Twenty-one patients with various oral lesions were enrolled in the study. The lesions were visualized using Identafi ${ }^{\circledR}$ device with white light illumination, followed by visualization of tissue autofluorescence and tissue reflectance. Tissue biopsied was obtained from the all lesions and both histopathological and immunohistochemical studies using a vascular endothelial biomarker (CD34) were performed on these tissue samples. The clinical vascular grading using the green-amber light at $545 \mathrm{~nm}$ and the expression pattern and intensity of staining for CD34 in the different biopsies varied depending on lesions, grading ranged from 1 to 3. The increase in vascularity was observed in abnormal tissues when compared to normal mucosa, but this increase was not limited to carcinoma only as hyperkeratosis and other oral diseases, such as lichen planus, also showed increase in vascularity. Optical spectroscopy is a promising technology for the detection of oral mucosal abnormalities; however, further investigations with a larger population group is required to evaluate the usefulness of these devices in differentiating benign lesions from potentially malignant lesions.
\end{abstract}

International Journal of Oral Science (2014) 6, 162-167; doi:10.1038/ijos.2014.39; published 25 July 2014

Keywords: angiogenesis; optical spectroscopy; oral lesions

\section{INTRODUCTION}

According to data from the American Cancer Society, an estimated 41380 new cases of cancer of the oral cavity and pharynx were expected in 2013, leading to an estimated 7900 deaths. ${ }^{1}$ The overall 5 -year survival rates for oral cancer have remained low at approximately $50 \%$ for the past decades and are considered among the worst of all cancer death rates including those for colorectal, cervix and breast origins. This is in part due to the lack of training of health professionals for early detection of premalignant/malignant lesions. Despite significant advances in cancer treatment, early detection of oral cancer and its curable precursors remains the best way to ensure patient survival and improved quality of life. ${ }^{2}$

It is well established that virtually all oral squamous cell carcinomas are preceded by visible changes in the oral mucosa, usually by way of white (leukoplakia) and red (erythroplakia) patches. ${ }^{1-3}$ In addition, there are other inflammatory disorders of the oral mucosa, such as lichen planus and submucous fibrosis that have been associated with an increased risk of oral squamous cell carcinoma development, designating these conditions as precancerous lesions. ${ }^{4}$ Furthermore, it is well documented that capillary changes precede tumor growth, with a pattern of tumor angiogenesis that is different from the usual neovascularization seen during repair and regeneration processes. ${ }^{5-8}$ Direct optical visualization of these patterns would be helpful in early determination of the underlying pathology and can also aid in determining the site for biopsy. ${ }^{9}$

Recent advancements in oral cancer research have led to the development of potentially useful diagnostic tools at the clinical and molecular level for the early detection of oral cancer. The gold standard for oral cancer diagnosis remains tissue biopsy with pathological assessment, but this technique needs a trained health care provider, and is considered invasive, painful, expensive and time consuming. Recently, scientific research in the field of oral cancer has focused on finding alternative approaches to traditional biopsy with emphasis on multifocal imaging devices. Depending on the type of light and the imaging approaches used, optical imaging of the oral tissues can detect minimal changes within the tissues, such as alterations in tissue architecture and composition,

${ }^{1}$ Section of Oral Medicine and Orofacial Pain, Division of Oral Biology and Medicine, School of Dentistry, University of California at Los Angeles, Los Angeles, USA; ${ }^{2}$ Section of Oral

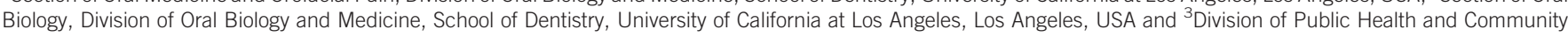
Dentistry, School of Dentistry, University of California at Los Angeles, David Geffen School of Medicine and Fielding School of Public Health, Los Angeles, USA

Correspondence: Dr DV Messadi, Section of Oral Medicine and Orofacial Pain, Division of Oral Biology and Medicine, School of Dentistry, University of California at Los Angeles, 10833 LeConte Avenue, CHS 63-019, Los Angeles CA 90095, USA

E-mail: dmessadi@dentistry.ucla.edu

Accepted 3 June 2014 
expression of specific biomarkers and vascularity/angiogenesis. The most common devices that have been marketed to dentists include: ViziLite ${ }^{\mathrm{TM}}$ (Zila, Batesville, AR, USA), VELscope ${ }^{\mathrm{TM}}$ (LED Dental, Vancouver, Canada), DIFOTI ${ }^{\mathrm{TM}}$ (Electro-Optical Sciences, Irvington, NY, USA) and Identafi ${ }^{\circledR}$ (StarDental-DentalEZ, Lancaster, PA, USA).

In the past decade, several forms of autofluorescence technology have been developed for inspection of the oral mucosa. Velscope and Identafi optical devices are the two most common devices used by general dentists. Fluorophores within the oral epithelium and stroma absorb ultraviolet and visible light and can re-emit some of this light at longer wavelengths in the form of fluorescence. Autofluorescence originates from a variety of fluorophores in the oral cavity and is sensitive to alterations in both tissue morphology and biochemistry associated with neoplasia. ${ }^{10-12}$ Oral cancer and precancer display a loss of autofluorescence across a broad range of ultraviolet and visible excitation wavelengths; as described later, this loss of fluorescence is largely attributed to a decrease in fluorescent crosslinks associated with stromal collagen that underlies the neoplastic lesions. ${ }^{13-15}$ Oral cancer is also associated with increased angiogenesis, which can affect both autofluorescence and reflectance spectra. In addition to combining the anatomical imaging with fluorescence, fiber optics and confocal microscopy, the Identafi technology with green-amber light (540- to 575-nm wavelength) illumination also examines the tissue reflectance to detect changes in angiogenesis. Reflectance spectroscopy uses light within the absorption spectrum of hemoglobin, in the range between 400 and $600 \mathrm{~nm}$-to visualize the underlying vasculature. ${ }^{15-18}$ Angiogenic vasculature in tumors differs from that in healthy tissue in several respects. Chronic overproduction of pro-angiogenic factors in tumors leads to uncontrolled development of new blood vessels. As a result, the number of blood vessels within a given microscopic area (i.e., microvessel density, MVD) is usually high but rarely uniform. ${ }^{8,19}$

The assessment of tissue angiogenesis in oral mucosal lesions with the green-amber light enables the clinician to correlate MVD identified by specific endothelial cell markers with clinicopathological parameters. ${ }^{18}$ The reduction in the reflectance spectra of oral cancer and precancerous lesions has been shown to occur around $577 \mathrm{~nm}$ and $542 \mathrm{~nm}$, and this is could be due to the increase in light absorption from the enhancement of microvasculature density and oxygenated hemoglobin concentrations in neoplastic tissue. ${ }^{16-18}$ Angiogenesis is an early step in carcinogenesis and a significant increase in microvessel count occurs in mild and moderate dysplasia. ${ }^{19-20}$ Studies have shown that tumor-induced angiogenesis results in altered vascular morphology, and the degree of change can assist with determining the prognosis of oral lesions. ${ }^{21-22}$ This suggests that assessment of tissue angiogenesis in oral mucosal lesions enables the clinician to differentiate dysplastic lesions from benign ones. To date, however, there are very few published reports regarding the use of the $545 \mathrm{~nm}$ greenamber light. $^{17-18}$

The aim of the current pilot study is to correlate the clinical grading of tissue vascularity in a variety of oral mucosal lesions, including leukoplakias and erythroplakias using the Identafi system with the histological grading of the biopsied lesions using specific vascular markers.

\section{MATERIALS AND METHODS}

The device used to screen the oral suspicious lesions for vascular changes was the Identafi ${ }^{\circledR}$ system (StarDental-DentalEZ, Lancaster, PA, USA). This portable tool has a light source in the form of a probe-like device that resembles a dental mirror and can, following appropriate infection control methods, be easily inserted into the mouth for oral examination. The amber light is designed to enhance the reflective properties of the oral mucosa, allowing a distinction between normal and abnormal tissue vasculature. ${ }^{18}$

\section{Study population}

Study subjects (21) were enrolled in a clinical protocol reviewed and approved by the Institutional Review Boards at The University of California (Los Angeles, CA, USA). Patients were eligible and recruited if they were 18 years of age or older and had a suspicious lesion in the oral cavity that needed to be biopsied, these lesions included, leukoplakia, erythroplakia, lichen planus and nonspecific ulceration. All subjects enrolled in the study gave written informed consent.

\section{Imaging procedures}

The Identafi ${ }^{\circledR}$ device has three light sources that can be used for the clinical examination: a white light for regular illumination, a violet light that excites fluorescence at $405 \mathrm{~nm}$ for tissue absorption, and a greenamber light at $545 \mathrm{~nm}$ for tissue reflectance. Reflectance spectroscopy uses light within the absorption spectrum of hemoglobin-namely, between 400 and $600 \mathrm{~nm}$ - to visualize the underlying vasculature. ${ }^{14-17}$ Patients recruited for the study were clinically assessed for their oral lesion by two oral medicine practitioners using the device's white light illumination and baseline photographs were obtained using a Nikon 4000 intra-oral camera. Briefly, the Identafi ${ }^{\circledR}$ system probe was used to shine light in the mouth showing the entire field of view. A mirror was attached to the probe to help visualize areas behind the teeth and in the pharynx. The suspicious lesions were visualized and photographed by using first the white light, the tissue autofluorescence and then the tissue reflectance light settings. Measurements were performed in a darkened room to minimize the effects of ambient light. All images were reviewed three times, by the two oral medicine clinicians that were blinded to the histology. The clinical diagnoses of the lesions selected ranged from erythroplakia, leukoplakia, and lichenoid reactions to non-specific nodular, vascular and pigmented lesions.

Clinical grading of the microvascular parameters includes the number of mucosal blood vessels and the average microvessel diameter were semiquantitavely assessed independently by two oral medicine specialist and given a scale of 1-3 under the $545 \mathrm{~nm}$ light, with 3 given to the most intense vascularity observed, considering both the number and architecture of blood vessels in the oral lesions, and 1 having the least number of microvasculature. ${ }^{21-23}$ Upon completion of the optical measurements, tissue biopsies were obtained from the lesions by using either scalpel or punch technique under local anesthesia and samples were submitted for histopathological assessment.

\section{Histopathological correlation}

Biopsies and resected tissues were evaluated using standard histopathological analysis by a board-certified oral pathologist who was blinded to the clinical diagnosis. Histopathology diagnosis of the oral lesions included the following categories: hyperkeratosis, lichenoid reaction, squamous cell carcinoma, dysplasia and other oral diseases as described later.

\section{Angiogenic marker}

A commonly used endothelial cell marker CD34 was utilized to identify positive stained endothelial cells. ${ }^{24-25}$ Briefly, 4- $\mu \mathrm{m}$ paraffin sections from the resected tissue paraffin blocks were deparaffinized in xylol and rehydrated in graded alcohol series. Endogenous peroxidase was inhibited using $3 \% \mathrm{H}_{2} \mathrm{O}_{2}$ in methanol. The sections were then washed in distilled water and heated in a microwave oven (in citrate buffer $10 \mathrm{mmol} \cdot \mathrm{L}^{-1}, \mathrm{pH}$ 6) for $15 \mathrm{~min}$ for epitope retrieval. Monoclonal 
Table 1 Patient demographics

\begin{tabular}{|c|c|c|c|c|c|c|}
\hline Gender & Age & Tobacco & Alcohol & CD34 grading & Clin grade & Clinical diagnosis \\
\hline $\mathrm{F}$ & 78 & $\mathrm{~N}$ & $\mathrm{~N}$ & 3 & 1 & Leukoplakia \\
\hline M & 51 & Y & Y & 3 & 3 & Squamous cell carcinoma \\
\hline M & 81 & Y & Y & 2 & 2 & Proliferating verrocous leukoplakia \\
\hline M & 53 & Y & Y & 2 & 2 & Leukoplakia \\
\hline M & 71 & $\mathrm{~N}$ & $\mathrm{~N}$ & 3 & 3 & Lichen Planus \\
\hline $\mathrm{F}$ & 71 & $\mathrm{~N}$ & N & 3 & 3 & Leukoplakia \\
\hline M & 73 & Y & $\mathrm{N}$ & 1 & 1 & Leukoplakia \\
\hline M & 56 & $\mathrm{~N}$ & N & 3 & 3 & Squamous papilloma \\
\hline $\mathrm{F}$ & 54 & Y & Y & 1 & 1 & Leukoplakia \\
\hline $\mathrm{F}$ & 72 & Y & N & 2 & 2 & Leukoplakia \\
\hline M & 71 & N & N & 2 & 3 & Lichen planus \\
\hline M & 45 & Marijuana & Y & 3 & 3 & Leukoplakia \\
\hline M & 47 & $\mathrm{~N}$ & $\mathrm{~N}$ & 3 & 2 & Peripheral ossifying fibroma \\
\hline M & 84 & $\mathrm{~N}$ & $\mathrm{~N}$ & 1 & 1 & Proliferating verrocous leukoplakia \\
\hline M & 53 & Y & $\mathrm{N}$ & 3 & 3 & Leukoplakia \\
\hline M & 32 & N & Y & 2 & 2 & Leukoplakia \\
\hline M & 20 & $\mathrm{~N}$ & $\mathrm{~N}$ & 2 & 2 & Peripheral ossifying fibroma \\
\hline $\mathrm{F}$ & 63 & $\mathrm{~N}$ & Y & 1 & 2 & Melanotic macule \\
\hline M & 55 & N & $\mathrm{N}$ & 2 & 3 & Vascular malformation \\
\hline M & 49 & Marijuana & $\mathrm{N}$ & 1 & 2 & Leukoplakia \\
\hline M & 62 & N & $\mathrm{N}$ & 1 & 3 & Squamous cell carcinoma \\
\hline
\end{tabular}

F, female; $M$, male.

CD 34: antibody for detection of endothelial cells.

Clinical grade: clinical grading using the angiogenic amber light probe.

antibodies against CD34 (Santa Cruz Biotechnology, Santa Cruz, CA, USA) were used and immunohistochemistry was performed using standard techniques. For negative control, sections were treated as above but without the primary antibody. Antibody binding was revealed using $\mathrm{H}_{2} \mathrm{O}_{2}$ as a substrate and diaminobenzidine as chromogen. A standard approach for quantifying angiogenesis in tissue sections is the measurement of MVD maximal number of blood vessels per unit area of section. ${ }^{26}$ Used in hundreds of studies, microvascular density is a significant prognostic indicator in many cancers.

\section{Vascular quantification}

MVD was estimated using a light microscope (Zeiss Axioscope; Zeiss, Oberkochen, Germany) by counting the microvessels at 200 magnification as reported by Weidner (1995). ${ }^{26}$ The immunostained sections for CD34 were carefully scanned at low magnifications, $\times 4$ and $\times 10$ objective lens, thereby finding three separately located lesion areas, where the highest number of discrete microvessels were stained (hot spots). Each hot-spot area was equivalent to a high power field with a $\times 20$ objective lens and field diameter $0.50 \mathrm{~mm}$. The vascular grading is both influenced by the number of vessel profiles in the initial scanning for hot-spots and by the area of the vessel profiles within the hot spots in the successive grading process. Vessels were counted in three hot spots areas and were assessed in a blinded fashion by two investigators independently. Depending on the number of positive vessels, lesions were graded as group 1 (fewer than 25 vessels/field); group 2 (25-75 vessels/field) and group 3, over 75 vessels per field. ${ }^{24,26}$ The determination of angiogenesis was performed without knowledge of the clinical vascular grading. There were no training used sets to correlate the clinical grade to the CD34 histology; actually both the two clinical graders and the histopathological grader were blinded from each other's grading so not to bias their results.

Data and statistical analysis

Distributions of demographics (age and gender), tobacco use, alcohol use, CD34 MVD grading and clinical grade, as well as lesion diagnosis, were calculated. The correlation between CD34 grading and clinical grading was evaluated through $2 \times 2$ concordance table and correlation coefficient. SAS Statistical Software V9.4 was used for all statistical analysis.

\section{RESULTS}

Patients were evaluated by direct visualization of the oral cavity with the white light, the violet light-excited fluorescence $(405 \mathrm{~nm})$ and the green-amber reflected light $(545 \mathrm{~nm})$. The patients experienced no harmful or adverse side effects from the screening. For the 21 patients included in the study, the mean age was 59 years (standard deviation 16) with a range from 20 to 84 years. The data were collected from September 2011 to October 2012. The majority of the subjects were male $(n=16,76 \%)$ with female being $24 \%(n=5)$. Ten $(48 \%)$ out of the 21 patients had a history of recent or past tobacco use (Table 1).

Table 2 shows the histopathological diagnosis for the oral mucosal lesions in this study. Among the 21 cases, nine lesions were diagnosed as hyperkeratosis, five lesions were either dysplastic or squamous cell carcinoma, two were lichenoid reaction and the remainder had other benign diagnoses (see Table 2 for the complete distribution of diagnosis list). Figures 1 and 2 show the difference with the green-amber

\section{Table 2 Histopathological diagnosis of biopsied cases}

\begin{tabular}{lcc}
\hline Histopathological diagnosis & Frequency & Percentage/\% \\
\hline Leukoplakia/hyperkeratosis & 9 & 42.86 \\
Lichenoid reaction & 2 & 9.52 \\
Leukoplakia/dysplasia & 1 & 4.76 \\
Proliferating verrocous leukoplakia & 2 & 9.52 \\
Squamous cell carcinoma & 2 & 9.52 \\
Vascular proliferation (HH8-ve) & 1 & 4.76 \\
Focal melanosis & 1 & 4.76 \\
Peripheral ossifying fibroma & 2 & 9.52 \\
Squamous papilloma & 1 & 4.76 \\
\hline
\end{tabular}



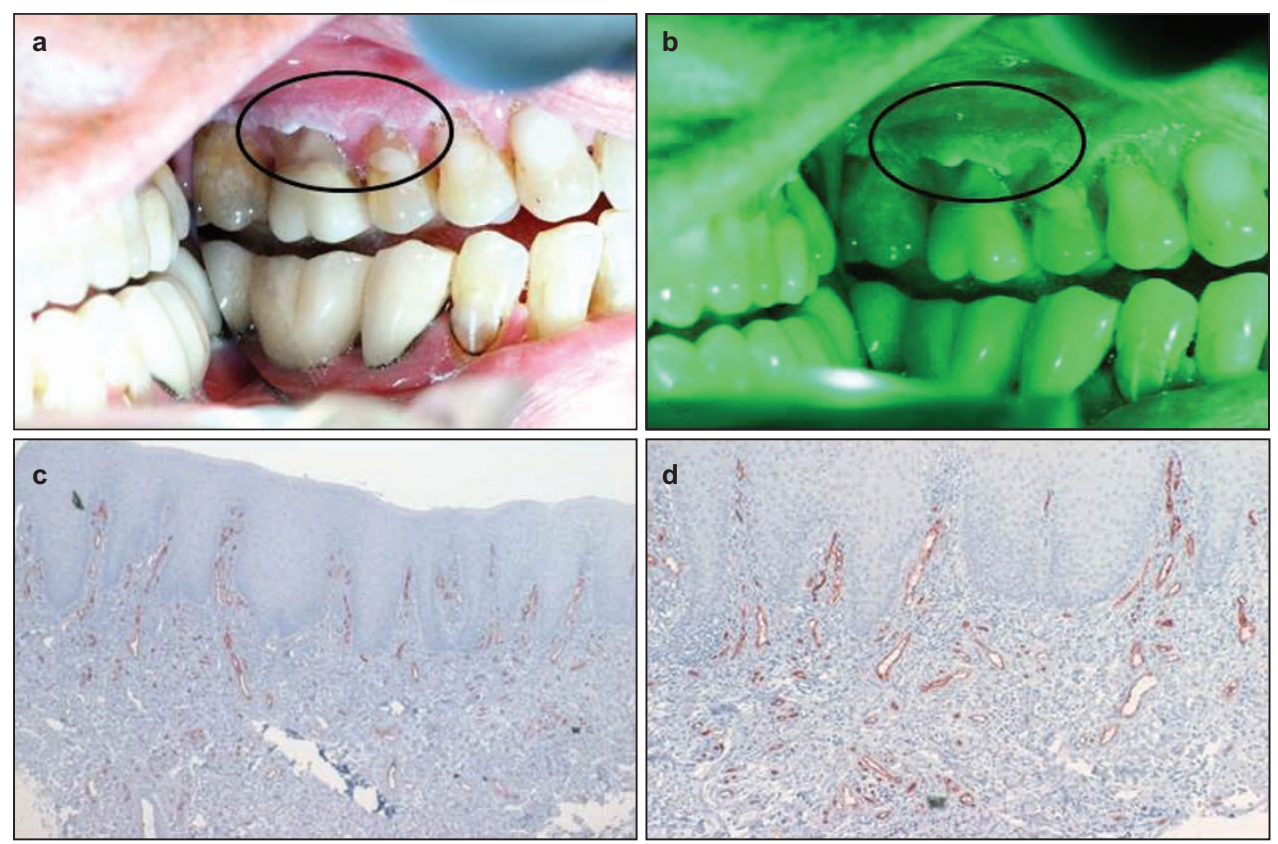

Figure 1 Leukoplakia/hyperkeratosis lesion showing decreased vascularity. (a) Picture of a proliferating verrucous leukoplakia on the left upper gingival tissue of an 84-year-old male shows clinical picture of the lesion taken with the white reflectance (regular light) Identafi® StarDental optical device. (b) Application of the Identafi® system with the green amber reflectance light show low number of blood vessels of the suspicious areas, where the red small blood vessels picture is not prominent. (c) Immunohistochemical expression of CD34, note low number of positive brown staining blood vessels ( $\times 40)$; (d) the same tissue at $\times 200$. A biopsy taken from this area showed hyperkeratosis with no dysplasia.

light at $545 \mathrm{~nm}$ where grade 3 hyperkeratosis (Figure 2a) shows enhanced vascularity under the light (Figure $2 \mathrm{~b}$ ) and increased vascularity (Figure $2 \mathrm{c}$ and $2 \mathrm{~d}$ ) when compared to a similar hyperkeratosis diagnosis of Grade 1 (Figure 1) with decrease in number of blood vessels. The

same Grade 3 intense vascular staining was also observed in a case of squamous cell carcinoma (Figure 3 ).

The expression pattern and number of positive vessels for CD34 in the different biopsies varied depending on the lesions diagnoses with
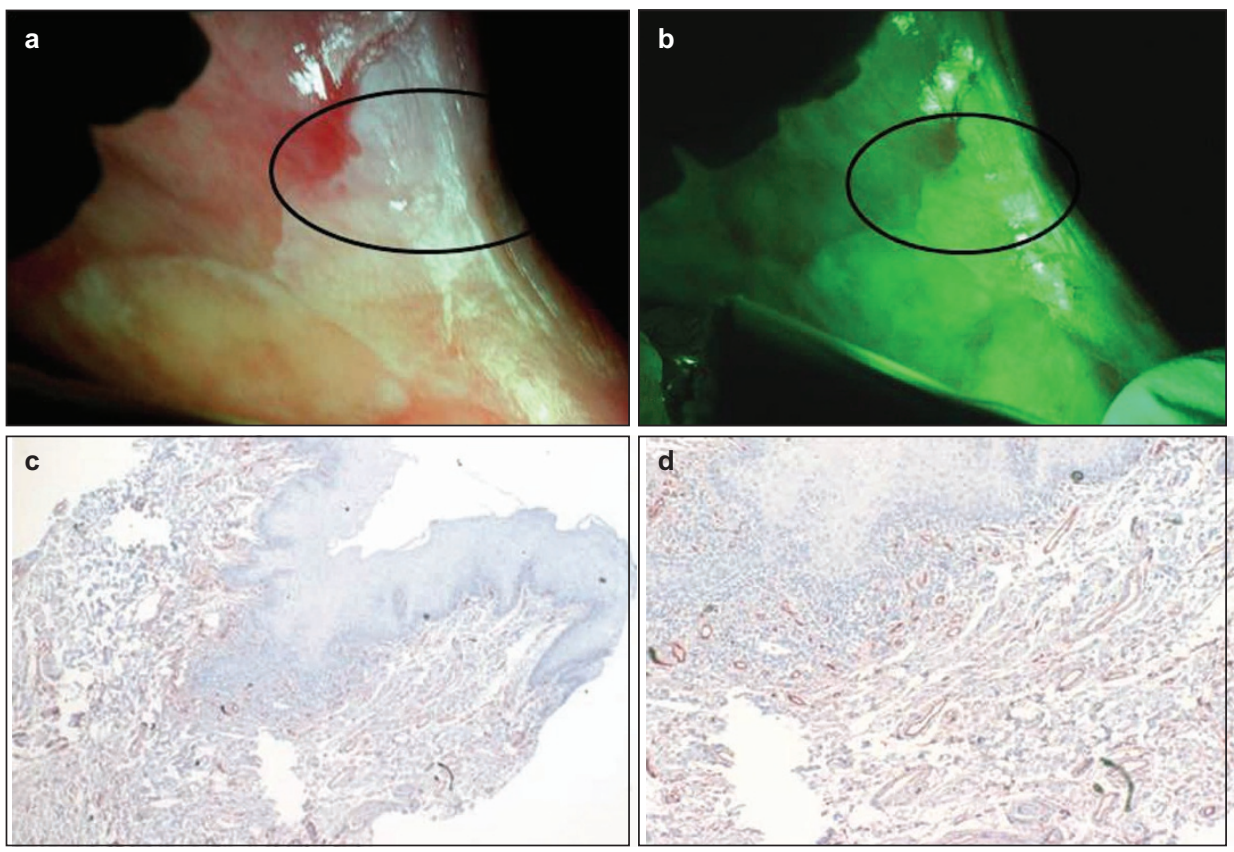

Figure 2 Leukoplaikia/hyperkeratosis lesion showing enhanced vascularity. (a) Picture of a leukoplakia lesion on the left buccal mucosa of a 53-year-old male patient taken with the white reflectance (regular light) Identafi ${ }^{\circledR}$ StarDental optical device. (b) Application of the Identafi ${ }^{\circledR}$ system with the green amber reflectance light at $545 \mathrm{~nm}$ enhanced visualization of the keratinized lesions over that of the white light image shown in (a), thus enhancing the superficial vasculature and demonstrating an increase in blood vessels number in the leukoplakia/hyperkeratotic lesion. (c) Immunohistochemical expression of CD34, note the high number of intense brown staining of positive blood vessels ( $\times 40)$; (d) the same tissue at $\times 200$. A biopsy taken from this area showed hyperkeratosis with mild inflammation and no dysplasia. 

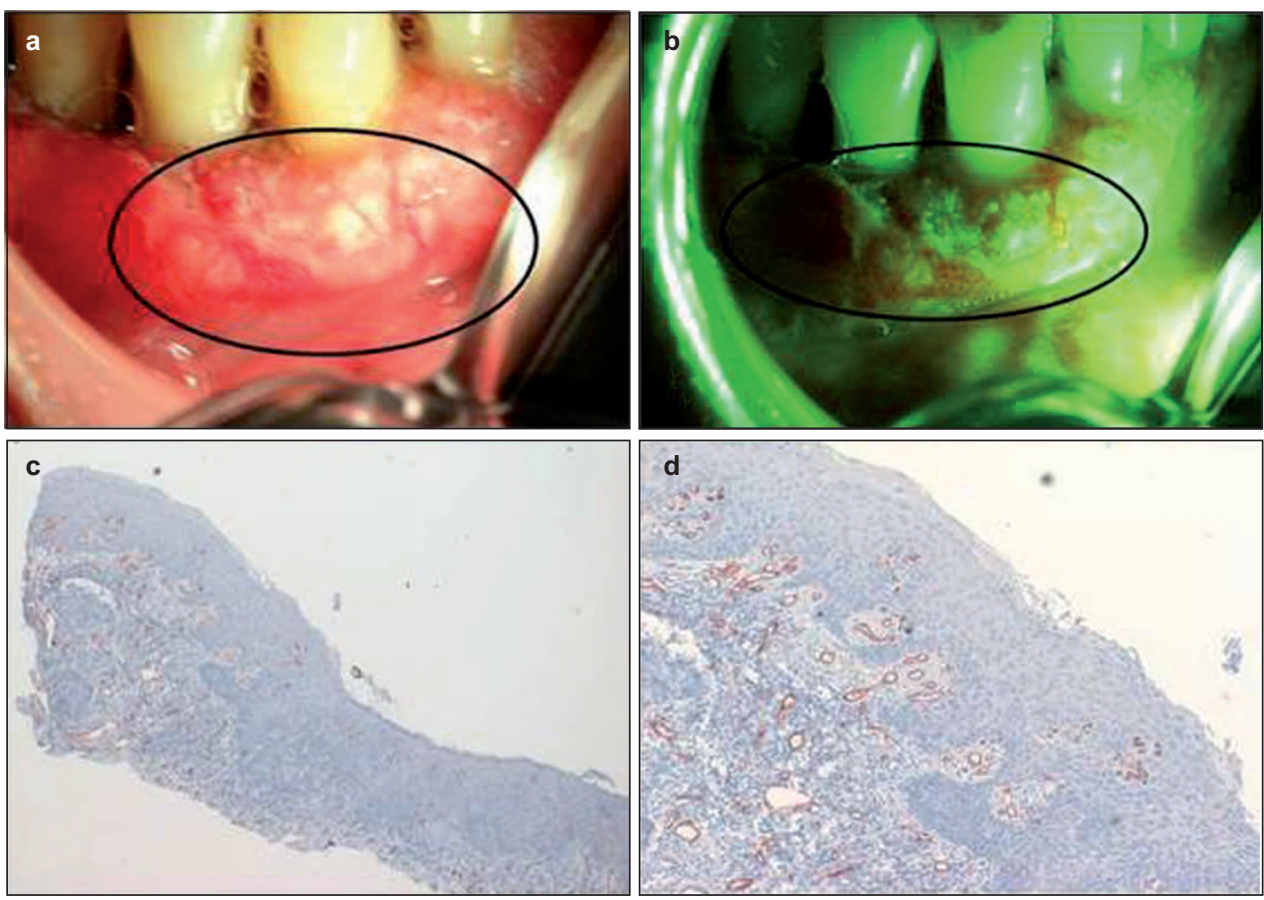

Figure 3 Squamous cell carcinoma lesion showing enhanced vascularity. (a) Picture of an erythroplakia lesion on the lower gingiva of a male patient taken with the white reflectance (regular light) Identafi ${ }^{\circledR}$ StarDental optical device. (b) Application of the Identafi ${ }^{\circledR}$ system with the green amber reflectance light at $545 \mathrm{~nm}$ show increase vascularity in the squamous cell carcinoma lesion demonstrated by increase in the number of red blood vessels observed. (c) Immunohistochemical expression of CD34, note the high number of intense brown staining positive blood vessels ( $\times 40)$; (d) the same tissue at $\times 200$. A biopsy taken from this area showed a moderately differentiated squamous cell carcinoma.

Table 3 Angiogenic correlation between histology (CD 34) and clinical grading

\begin{tabular}{llcl}
\hline \multirow{2}{*}{ CD34 grading } & \multicolumn{3}{c}{ Clinical grade } \\
\cline { 2 - 4 } & 1 & 2 & 3 \\
\hline 1 & 3 & 2 & 1 \\
2 & 0 & 5 & 2 \\
3 & 1 & 1 & 6 \\
\hline
\end{tabular}

grading ranging from $1-3$ (Figures $1 \mathrm{~d}, 2 \mathrm{~d}$ and $3 \mathrm{~d}$ ). Table 3 shows the distribution of concordant/discordant relationship between clinical grading and CD34 grading for the study patients. Out of the 21 patients, $14(66 \%)$ had perfect agreement between CD34 and clinical grading. The overall correlation between CD34 and clinical grading was 0.5155 (95\% confidence interval: $0.1218-0.9091$ ). It should be noted that the increase in vascularity was observed in abnormal tissues when compared to normal mucosa but this increase was not limited to carcinoma only (Figure 3 and Table 1), as leukoplakia/hyperkeratosis (non-cancerous lesions) and other oral diseases, also showed increase in vascularity (Figure 2 and Table 1).

\section{DISCUSSION}

Several studies have recently been published on the use of tissue reflectance for diagnosis of suspicious lesions in several organs, including a recent study using Identafi ${ }^{\circledR}$ for screening of 124 subjects. This study demonstrated a sensitivity of $82 \%$ and a specificity of $87 \%$ in differentiating between neoplastic and non-neoplastic oral conditions. The study results appeared to vary between sampling depths, and keratinized $v s$. non-keratinized tissues. ${ }^{18}$ To date, however, there are very few published reports regarding the use of the $545 \mathrm{~nm}$ green-amber light. ${ }^{16,27}$ Tissue reflectance setting in these multispectral devices is based on the premise of detecting changes in angiogenesis with green-amber light (540- to 575-nm wavelength) illumination. While the white light examination is performed first as the standard of care for the visual examination of the suspicious lesions, examination with the Identafi ${ }^{\circledR}$ system amber light is subsequently used to distinguish between normal and abnormal tissue vasculature. ${ }^{28-29}$

In this pilot study, we are the first to correlate the tissue reflectance that detects vascular changes clinically with histological vascularity in various oral lesions. In this small sample size study, the clinical tissue reflectance angiogenesis grading significantly correlated with histological vascularity grading; using the angiogenic marker CD34, this biomarker is mainly used to identify the positive endothelial cells and to quantify vascularity in tissue sections. ${ }^{25}$ In addition, our findings confirmed that the $545 \mathrm{~nm}$, the green-amber light enhanced the clinical appearance of the keratinization and the vascularity of the lesions, making them larger and more visible to the naked eye than the regular white light. ${ }^{18}$ This visual enhancement of the lesion vascularity was observed in abnormal tissues when compared to normal mucosa. However, the increase in vascularity was not limited to carcinoma only, as leukoplakia/hyperkeratosis (non-cancerous lesions) and other oral diseases such as lichen planus that had an inflammatory component, also showed increase in vascularity. Whether leukoplakia/ hyperkeratosis lesions with grade 3 vascularity had a higher potential for malignant transformation is not clear from this study and may only be determined with long term follow-up of these patients.

In this study, from a point of care perspective, non-invasive examination with the Identafi system enhanced the clinical assessment and confirmed the clinician's suspicion of increased vascularity in the oral mucosal lesions. Therefore, patients that demonstrated a grade 3 on both, the clinical and the histological vascular grading, were assigned 
for more frequent periodic clinical assessment and careful long term follow-up. However, to truly establish the magnitude of this methodology's contributions to early detection of precancerous and cancerous conditions, larger well-designed prospective studies are required.

Optical spectroscopy is a promising technology for the diagnosis of oral neoplasia. As further research into the biological mechanisms of angiogenesis associated with oral cancer continues, a more clear understanding of the microvascular changes in oral premalignant lesions and oral cancers will advance our understanding of the oral carcinogenic process. ${ }^{8,19-20}$ That knowledge coupled with further studies of the molecular basis for fluorescence imaging will help determine those factors that contribute to false-positive and false-negative results in using these imaging modalities.

\section{CONCLUSION}

The results of this pilot study are very encouraging, but further investigations with a larger population group is needed to establish definitively, whether this screening device significantly increases the assessment sensitivity in detecting precancerous lesions over comprehensive white light examination and to validate the efficacy of this screening device for use by general dentists.

\section{CONFLICT OF INTEREST}

This study was funded by a research gift from StarDental-DentalEZ, Lancaster, PA, USA.

\section{ACKNOWLEDGEMENTS}

We are grateful to Dr Russell Christensen for his pathological diagnosis of our biopsy specimen. We would like to thank Mr Michael Gordon for his editorial support.

1 Kerr AR, Shah SS. Standard examination and adjunctive techniques for detection of oral premalignant and malignant lesions. J Calif Dent Assoc 2013; 41(5): 329-331, 334-342.

2 Messadi DV. Diagnostic aids for detection of oral precancerous conditions. Int J Oral Sci 2013; 5(2): 59-65.

3 Jemal A, Siegel R, Xu J et al. Cancer statistics, 2010. CA Cancer J Clin 2010; 60(5): 277-300.

4 Fedele S. Diagnostic aids in the screening of oral cancer. Head Neck Oncol2009; 1: 5 .

5 Folkman J, Watson K, Ingber D et al. Induction of angiogenesis during the transition from hyperplasia to neoplasia. Nature 1989; 339(6219): 58-61.

6 Hasina R, Lingen MW. Angiogenesis in oral cancer. J Dent Educ 2001; 65(11): 1282 1290.

7 Raica M, Cimpean AM, Ribatti D. Angiogenesis in pre-malignant conditions. Eur J Cancer 2009; 45(11): 1924-1934.

8 Pazouki S, Chisholm DM, Adi MM et al. The association between tumor progression and vascularity in the oral mucosa. J Pathol 1997; 183(1): 39-43.

9 Shetty DV, Ahuja P, Taneja DK et al. Relevance of tumor angiogenesis patterns as a diagnostic value and prognostic indicator in oral precancer and cancer. Vasc Health Risk Manag 2011; 7: 41-47.
10 Bouquot JE, Suarez P, Vigneswaran N. Oral precancer and early cancer detection in the dental office-review of new technologies. J Implant Adv Dent2010; 2(3): 47-63.

11 Epstein JB, Güneri $\mathrm{P}$, Boyacioglu $\mathrm{H}$ et al. The limitations of the clinical oral examination in detecting dysplastic oral lesions and oral squamous cell carcinoma. J Am Dent Assoc 2010; 143(12): 1332-1342.

12 Lingen MW, Kalmar JR, Karrison T et al. Critical evaluation of diagnostic aids for the detection of oral cancer. Oral Oncol 2008; 44(1): 10-22.

13 Rosin MP, Poh CF, Guillard M et al. Visualization and other emerging technologies as change makers for oral cancer prevention. Ann N Y Acad Sci 2007; 1098: 167-183.

14 Patton LL, Epstein JB, Kerr AR. Adjunctive techniques for oral cancer examination and lesion diagnosis: a systematic review of the literature. J Am Dent Assoc 2008; 139(7): 896-905.

15 Schwarz RA, Gao W, Daye D et al. Autofluorescence and diffuse reflectance spectroscopy of oral epithelial tissue using a depth-sensitive fiber-optic probe. Appl Opt 2008; 47(6): 825-834.

16 Zuluaga AF, Vigneswaran N, Bradley RK et al. Identafi, 3000 ultra a multispectral tool for improved oral lesion evaluation. Washington: Optical Society of America, 2010.

17 Bhatia N, Lalla Y, Vu AN et al. Advances in optical adjunctive aids for visualisation and detection of oral malignant and potentially malignant lesions. Int J Dent 2013; 2013: 1-17. DOI: 10.1155/2013/194029.

18 Lane P, Follen M, MacAulay C. Has fluorescence spectroscopy come of age? A case series of oral precancers and cancers using white light, fluorescent light at $405 \mathrm{~nm}$, and reflected light at $545 \mathrm{~nm}$ using the Trimira identafi 3000. Gender Med2012; 9(1 Suppl): S25-S35.

19 Raica M, Cimpean AM, Ribatti D. Angiogenesis in pre-malignant conditions. Eur J Cancer 2009; 45(11): 1924-1934.

20 Shetty DC, Ahuja P, Taneja DK et al. Relevance of tumor angiogenesis patterns as a diagnostic value and prognostic indicator in oral precancer and cancer. Vasc Health Risk Manag 2011; 7: 41-47.

21 Miller JC, Pien HH, Sahani D et al. Imaging angiogenesis: applications and potential for drug development. J Nat/ Cancer Inst 2005; 97(3): 172-187.

22 McDonald DM, Choyke PL. Imaging of angiogenesis: from microscope to clinic. Nat Med 2003; 9(6): 713-725.

23 Amelink A, Kaspers OP, Sterenborg HS et al. Non-invasive measurement of the morphology and physiology of oral mucosa by use of optical spectroscopy. Oral Oncology 2008; 44(1): 65-71.

24 Gonzalez-Palacios F, Sancho M, Martinez JC et al. Microvessel density, p53 overexpression, and apoptosis in invasive breast carcinoma. Mol Pathol 1997. 50(6): 304-309.

25 Pusztaszeri MP, Seelentag W, Bosman FT. Immunohistochemical expression of endothelial markers CD31, CD34, von Willebrand factor, and Fli-1 in normal human tissues. J Histochem Cytochem 2006; 54(4): 385-395.

26 Weidner N. Intratumor microvessel density as a prognostic factor in cancer. Am J Pathol 1995; 147(1): 9-19.

27 Sweeny L, Dean NR, Magnuson JS et al. Assessment of tissue autofluorescence and reflectance for oral cavity cancer screening. Otolaryngol Head Neck Surg 2011 . 145(6): 956-960.

28 Schwarz RA, Gao W, Weber CR et al. Noninvasive evaluation of oral lesions using depth-sensitive optical spectroscopy: simple device for the direct visualization of oral-cavity tissue fluorescence. Cancer 2009; 115(8): 1669-1679.

29 Schwarz RA, Gao W, Stepanek VMT et al. Prospective evaluation of a portable depthsensitive optical spectroscopy device to identify oral neoplasia. Biomed Opt Express 2011; 2(1): 89-99.

\section{(c) $($ This work is licensed under a Creative Commons Attribution-}

(c) $\mathrm{BY}$ NC ND NonCommercial-NoDerivs 3.0 Unported License. The images or other third party material in this article are included in the article's Creative Commons license, unless indicated otherwise in the credit line; if the material is not included under the Creative Commons license, users will need to obtain permission from the license holder to reproduce the material. To view a copy of this license, visit http://creativecommons.org/licenses/ by-nc-nd/3.0/ 